\title{
Symbol Timing Detection for OFDM Signals with Time Varying Gain
}

\author{
Jihye Lee and Taehyun Jeon ${ }^{\dagger}$ \\ Seoul National University of Science and Technology, \\ Dept. of Electrical and Information Engineering, \\ 232 Gongneung-ro, Nowon-Gu, 139-743 Seoul, Korea \\ \{wejior, thjeon\}@seoultech.ac.kr
}

\begin{abstract}
The packet based OFDM (Orthogonal Frequency Division Multiplexing) systems require the fast timing synchronization using a preamble located at the beginning of the received data frame. To detect the symbol timing of the received signal, periodicity of the preamble can be utilized. However, the time varying gain of preamble sample values can affect the performance of the synchronization in case the samples of the beginning part of the packet are involved. This is due to the AGC (Automatic Gain Control) operation at the receiver. Conventional symbol timing detectors use the very last part of the short preamble whose gain level is settled upon the completion of the AGC operation. In this paper the symbol timing detector is proposed using all parts of the short preamble to improve the detection performance keeping the independence of the AGC gain. Simulation results show that the proposed method shows compatible performance for various channel environments.
\end{abstract}

Keywords: OFDM, preamble, symbol timing detection, synchronization

\section{Introduction}

Recently, wireless data communication systems based on the OFDM (Orthogonal Frequency Division Multiplexing) physical layer become one of the major multimedia data transmission systems due to the advancement of the digital signal processing technology and its robustness to the multipath fading in the broadband channel environments. Some of the popular applications of the OFDM based system include IEEE802.11a/g/n and HIPERLAN/2 wireless local area network standards [1-3]. These standards have been developed to support the high-speed data network which can connect various types of mobile multimedia terminals including lab-top computers, tablet PCs, home entertainment devices, and even the smart phones in the range of tens to hundreds of meters. The OFDM scheme is suitable for the frequency selective fading channel since the symbol interval is relatively long compared with the single carrier case. This is possible since the OFDM utilizes the multi-carriers each of which transmits an independent data stream in parallel. The longer symbol interval provides the relative robustness to the multi-path effects which is equivalent to inter-symbol interference (ISI). The frequency selective fading due to the multi-path fading in the time domain can be easily compensated with one-tap equalizer in the frequency domain in OFDM receivers. While there exist many benefits in the OFDM system, the other aspects to be carefully considered are the synchronization errors due to the interferences including the oscillator instabilities and Doppler shifts.

$\dagger$ Corresponding Author 
The synchronization is an important issue in the OFDM systems which require the accurate estimation of the symbol timing and the carrier frequency offset. The errors in synchronization procedure may cause inter-carrier interference (ICI) and ISI which in turn results in the degradation of the overall system performance. The symbol timing detection process tries to find the optimum starting point of each OFDM symbol window. There are various types of timing detection methods for the fast and accurate symbol timing synchronization [4-5]. The preamble is one of the key timing information bearing samples which can be utilized for the synchronization. The repeated patterns of the preamble are useful characteristics for extracting carrier frequency and symbol timing offset. In IEEE802.11 based systems which are also known as WiFi, the preamble consists of ten short and two long cycles of preambles. The short preamble repeats with a period of 0.8 us in this system. The short preamble is designed for carrier detection, diversity selection, automatic gain control (AGC), carrier frequency offset estimation and symbol timing synchronization. Among ten periods, about seven periods of time are normally devoted to the first three operations mentioned above. In some conventional schemes only the remaining three periods are ready for the symbol timing detection. The first part (about 7 periods) may not be ready for the symbol timing detection depending on the method since the gain control is still on the way where the signal level is being varied. Some of the traditional methods for timing detection include auto-correlation and cross-correlation based method. With the autocorrelation method the fast synchronization can be obtained while relatively lower detection probability can be achieved in the low SNR (Signal-to-Noise Ratio) cases [6]. In this type of method the performance is also influenced by the preamble structure although the implementation is relatively simple using the periodicity of the preamble [7]. In an effort to overcome the problems of auto-correlation method the hybrid methods are proposed where both the cross and auto-correlation techniques are combined [8]. The power level of the received signal can also be utilized to further improve the performance of the hybrid method [6].

In general the improvement of performance comes with the increase of the system complexity. Also, the increase of the number of observation sample could improve the detection accuracy although there might exist conflicts with the AGC operation depending on the adopted algorithm. In this paper a new symbol timing detection method is proposed which offers a compatible performance in the time varying signal level environment. To achieve this goal, the proposed scheme adopts a one bit mapper where the available number of observation sample is maximized while maintaining the detection performance.

The organization of the paper is as follows. In the next section conventional symbol timing detection method is described with the effect of the AGC operation. In the following section the proposed gain independent detection scheme is discussed. The detection performances are also discussed in various channel environments including the SNR, delay spread and carrier frequency offset. Finally, concluding remarks are followed.

\section{Symbol Timing Detection based on Training Symbols}

In this section a conventional timing detection scheme is discussed in the time varying signal level environment. The discussion and the following simulation results are based on the IEEE 802.11a physical layer frame format although the method can be applied to any preamble based systems. The signal level of the beginning part of the receiver may fluctuate due to the AGC operation until it reaches the target gain value of the system. The varying gain stage normally continues for the duration of the first to seventh cycle of the short preamble. At this transient stage, the signal might be inaccurate due to the saturation and the 
quantization noise which can occur when the received signal mismatch with the dynamic range of the ADC (Analog-to-Digital Converter). Therefore the estimations are usually carried out based on the samples after the completion of the AGC which is equivalent to the eighth to the tenth cycle of short preamble symbols.

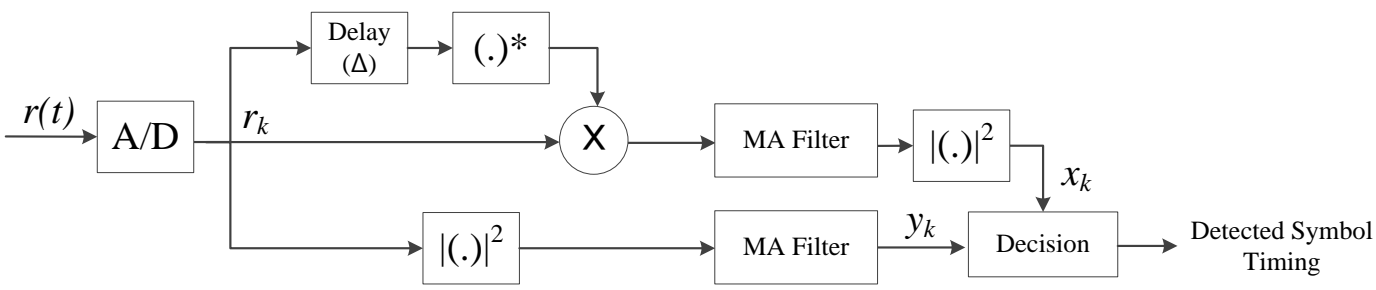

Figure 1. Block diagram of the conventional auto-correlation based detection scheme

The block diagram of the auto-correlation based detection scheme utilizing all short preamble sequences is shown in Figure 1. The auto-correlation is estimated between the received sample and its delayed version where the amount of delay $\Delta$ is set to 16 in this case. The required operations for the estimation include a complex multiplication, an absolute squaring and moving average filterings with a window size $W=9 \Delta$. The values of parameter $\Delta$ and $W$ are set such that a unique peak is generated at the output of the moving average filter. In this figure $x_{k}$ is the absolute squared value of the moving average filter output as shown in (1) [5]. Another moving average filter is used to measure the average power of the sample whose output is labeled as $y_{k}$ as described in (2). The measured power is used to normalize the correlation output to obtain the correlation coefficient $c_{k}$ which can be represented as in (3).

$$
\begin{gathered}
x_{k}=\left|\sum_{i=0}^{W-1} r_{k-i} r_{k-\Delta-i}^{*}\right|^{2} \\
y_{k}=\sum_{i=0}^{W-1}\left|r_{k-i}\right|^{2} \\
c_{k}=\frac{x_{k}}{y_{k}}
\end{gathered}
$$

The performance of the detector is shown in Figure 2 when the SNR is $24 \mathrm{~dB}$ and RMS (root-mean squared) delay spread is 50ns. We also assume that the AGC is on for the optimal signal level [9]. The ADC resolution is assumed to be 10-bit. Here the equivalent dynamic range of the $\mathrm{ADC}$ is assumed from -512 to 511. In order to analyze the accuracy of the timing acquisition, the statistics of the estimated position are measured. The performance analysis for the detector is carried out based on the measured CDF (Cumulative Distribution Function) of the detection error probability. Here the detection error is defined as the sample index differences between the actual and the detected symbol position. The CDF results of the conventional detector are shown in Figure 2. The relative convergence speed to the probability of one determines the detection performance. The faster convergence speed can be translated into the better performance. The result shows that this conventional scheme is not able to provide the acceptable detection performance even in the relatively high SNR case. The CDF does not converge to the probability of one even for the timing errors of 16 samples 
which is equivalent to the number of samples associated with the guard interval of the OFDM symbol. This means that this detector cannot be adopted for the case where the signal level of the samples are time varying.

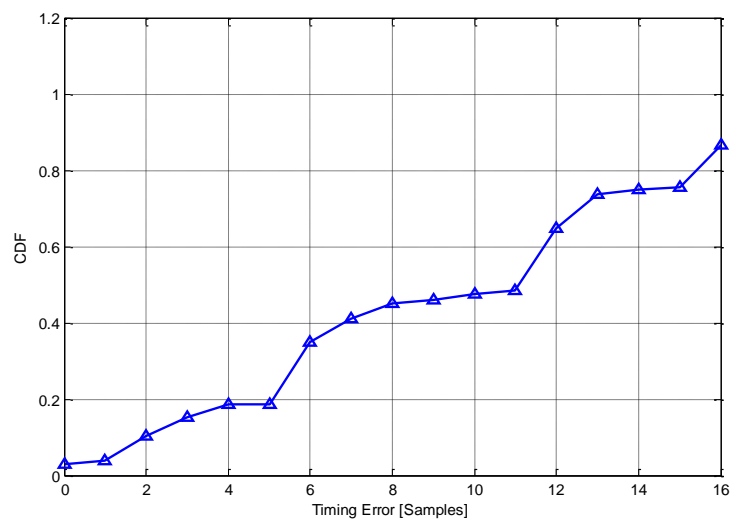

Figure 2. Performance of the conventional detection (with AGC on)

\section{Proposed Gain Independent Detector}

In this section a new proposed detection scheme is discussed to overcome the performance degradation due to the time varying signal level while maintaining the compatible detection performance. As discussed in the previous section the performance of the conventional autocorrelation based detector is very sensitive to the variation of the signal gain level. To reduce the effect of the AGC the one-bit mapper is placed after the ADC block. Here we assume that the ADC block is still required for other signal processing for the OFDM receiver. The hardware increase is not that significant due to the introduction of the one-bit mapper which requires one simple comparator. Even further the operation after the new inserted block becomes much simpler since the multiplications and the additions are now based on one-bit numbers. The block diagram of the proposed scheme is shown in Figure 3 [10]. As seen in the figure the structure is almost the same except the new insertion of a threshold detector. In this figure the power measurement block is excluded since the power level is always constant due to the 1-bit quantization. To confirm the effectiveness of the proposed scheme a comparative simulation is first conducted under the same channel condition as in the previous section. The simulation results are shown in Figure 4 in which the result of the previous section is redrawn in the same graph for comparison purposes. It can be observed a remarkable performance improvement with the proposed scheme. The simulation environments are the same as before ( $\mathrm{SNR}=24 \mathrm{~dB}$ and $\mathrm{RMS}$ delay spread=50ns).

More simulations are done to obtain the baseline performance of the proposed scheme under various types of noise environments. In this simulation the delay spread level is kept to $50 \mathrm{~ns}$ and the SNR of 12 and $24 \mathrm{~dB}$ is assumed. The results show that the detector performs well with acceptable sample position error statistics. As shown in Figure 5 the CDF curves are converged to the probability of one within 8 sample interval error for all ranges of SNRs tested. This baseline simulation results provides the upper bound performance of the proposed scheme which ultimately targets variable gain environments. 


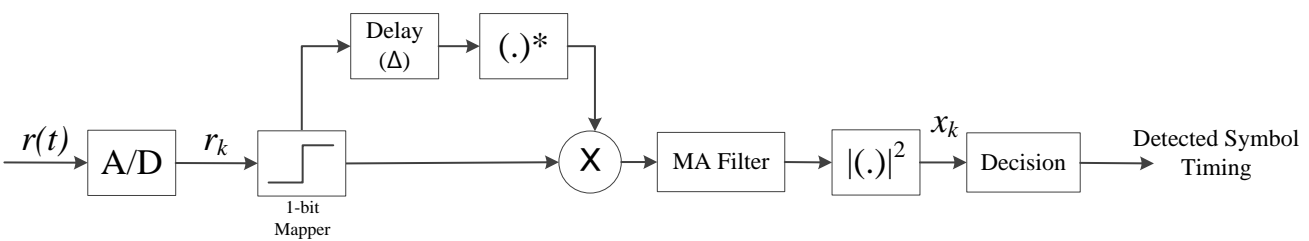

Figure 3. Block diagram of the proposed detection scheme

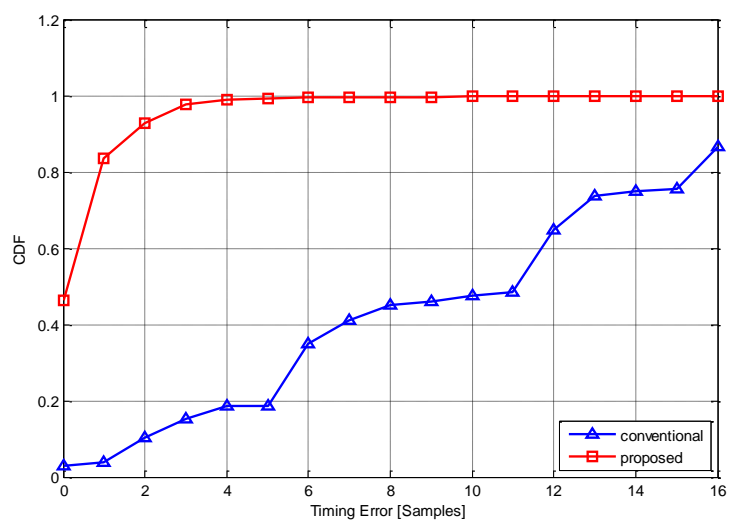

Figure 4. Performance comparison between conventional and proposed detector (with AGC on)

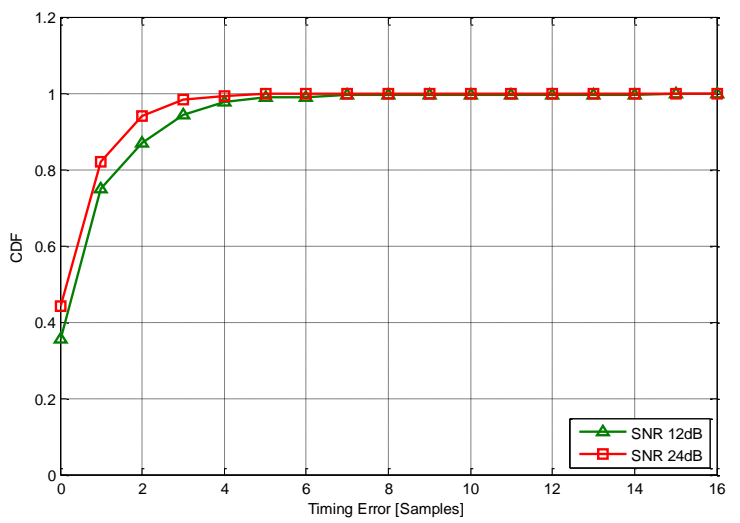

Figure 5. Performance of the proposed scheme for various noise levels (with AGC on)

To assess the effectiveness of the proposed scheme comparative simulation is performed. In this simulation we assume the extreme case of the gain level variation where the input to the ADC has very large signal level. This can cause the multiple adjustments of signal gain level through the AGC operation [9]. The other counterpart case assumes the optimal signal level for the given 10-bit ADC which results in no gain variation at all. In the varying gain case the initial input signal level to the ADC is assumed to be $70 \mathrm{~dB}$ which is quite big. In the other case the signal level is set to the optimal gain which is $29 \mathrm{~dB}$. The other channel parameters are set as SNR=24dB and RMS delay spread $=50 \mathrm{~ns}$ for two cases. The simulation 
results show the performance of the variable gain case shows very close to the base line curve which is the optimal fixed gain case. This result confirms the robustness of the proposed scheme to the gain variation of the preamble period.

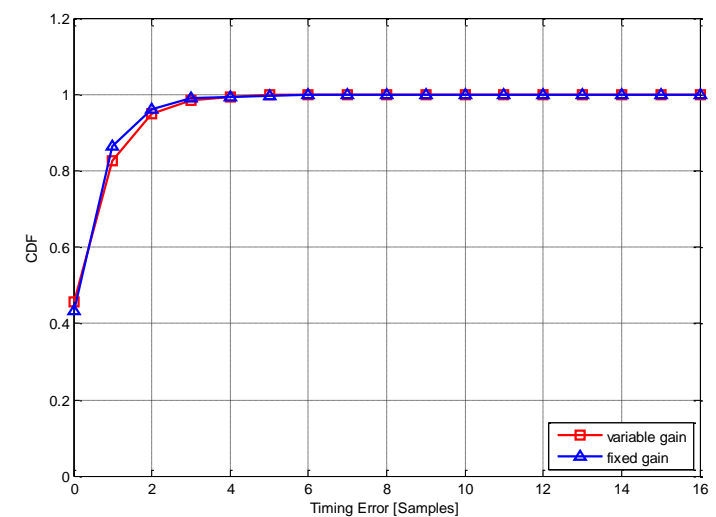

\section{Figure 6. Performance of the proposed detector for variable and constant signal level}

The effects of various amounts of delay spread are shown in Figure 7. For this simulation the SNR is assumed to be $24 \mathrm{~dB}$. As it can be expected the delay spread of the wireless channel affects the statistics of the detected position errors. Results show that more delay spread degrades the performance of the timing detector. Considering the guard interval duration of the system which is 800ns the performance of the proposed scheme is quite acceptable even under the influence of the delay spread.

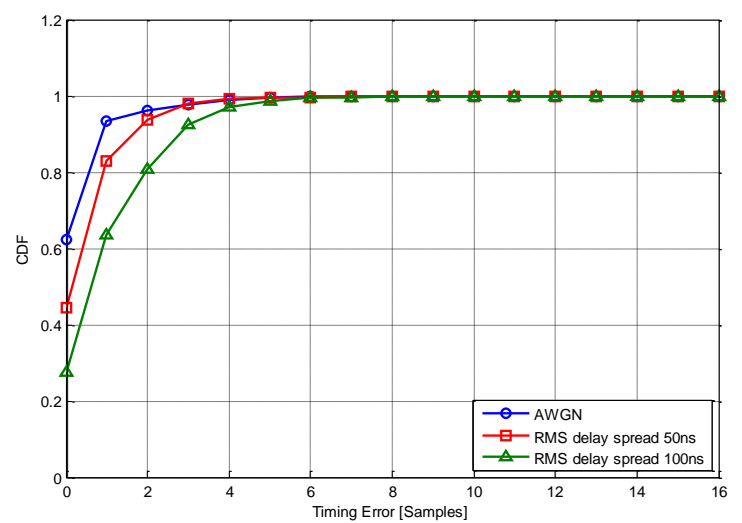

Figure 7. Performance of the proposed detector in multi-path channels

In order to measure the impact of the CFO (carrier frequency offset), another performance simulation is carried out assuming that RMS delay spread is 50ns and SNR is $24 \mathrm{~dB}$. The results are shown in Figure 8. The CFO estimation is normally carried out at the last stage of the short preamble. This indicates that the timing detection should be done based on the samples with carrier frequency offset errors. The maximum CFO which is allowed in the standard is $40 \mathrm{ppm}$. The results are shown in Fig. 8 where no CFO case and the maximum $\mathrm{CFO}$ case are compared. As shown in the CDF curves the performance degradation due to the 
frequency error is negligible. In other words the performance of the proposed timing detector is almost unaffected by the carrier frequency difference between the transmitter and the receiver.

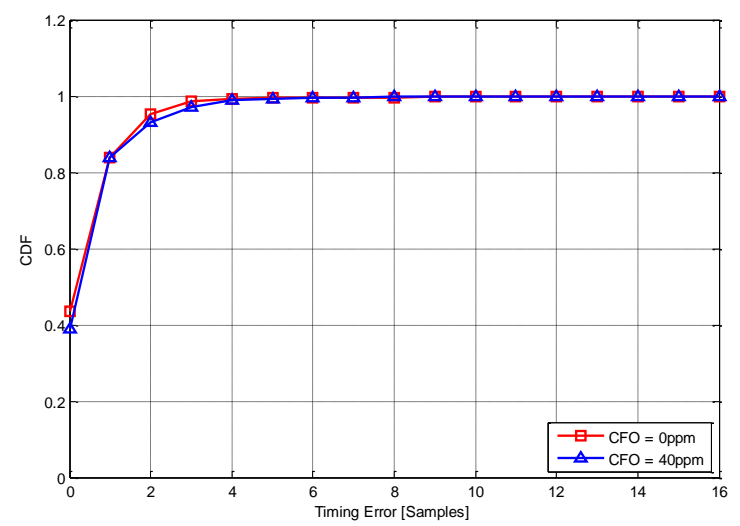

Figure 8. Performance of the proposed detector under the effect of CFO

\section{Conclusion}

In this paper, a symbol timing detector is proposed which is independent of the time varying signal gain. The advantage of the proposed scheme is the full utilization of available preamble symbols regardless of the stages of the AGC operation. The performance simulation is done in the IEEE 802.11a wireless local area network system. The proposed method combines auto-correlation and 1-bit mapping algorithms to solve the problem of the dependency of the signal gain. This detector tries to maximize the number of observation sample to improve the detection accuracy. At the same time the sensitivity to the variation of the signal level is minimized. The extensive simulations are carried out for various channel environments including SNR, delay spread and carrier frequency offset. The overall results show that the proposed scheme shows compatible performance even with the gain variation.

\section{References}

[1] IEEE Std., "Wireless LAN Medium Access Control (MAC) and Physical Layer (PHY) specification: High-speed Physical Layer in the 5GHz Band", IEEE Std 802.11a, (1999).

[2] IEEE Std., "Wireless LAN Medium Access Control (MAC) and Physical Layer (PHY) specifications: Further Higher Data Rate Extension in the 2.4 GHz Band", IEEE 802.11g, (2003).

[3] IEEE Std., "Wireless LAN Medium Access Control (MAC) and Physical Layer (PHY) specifications: Enhancements for Higher Throughput", IEEE 802.11n, (2008).

[4] J. K. Proakis, "Digital Communication", McGraw-Hill, New York, (2008).

[5] J. Terry and J. Heiskala, "OFDM Wireless LANs: A Theoretical and Practical Guide", SAMs, (2002).

[6] L. Zhou and M. Saito, "A new symbol timing synchronization for OFDM based WLANs under multipath fading channels", 15th IEEE International Symposium on Personal, Indoor and Mobile Radio Communications, vol. 2, (2004), pp. 1210-1214.

[7] S. K. Manusani and R. S. Kshetrimayum, "Robust Time and Frequency Synchronization in OFDM based 802.11a WLAN systems", Annual IEEE India Conference, (2006), pp. 1-4.

[8] S. Nandula and K. Giridhar, "Robust timing synchronization for OFDM based wireless LAN system", TENCON'03, (2003), pp.1558-1561.

[9] J. Lee and T. Jeon, "Optimization of Gain Control Loops for Packet Based Data Transmissions", International Journal of Control and Automation, vol. 6, no. 2, (2013), pp. 399-408.

[10] T. Jeon and S. Lee, "Symbol timing detector for wireless communication system", Korea Patent 100519917, (2005). 

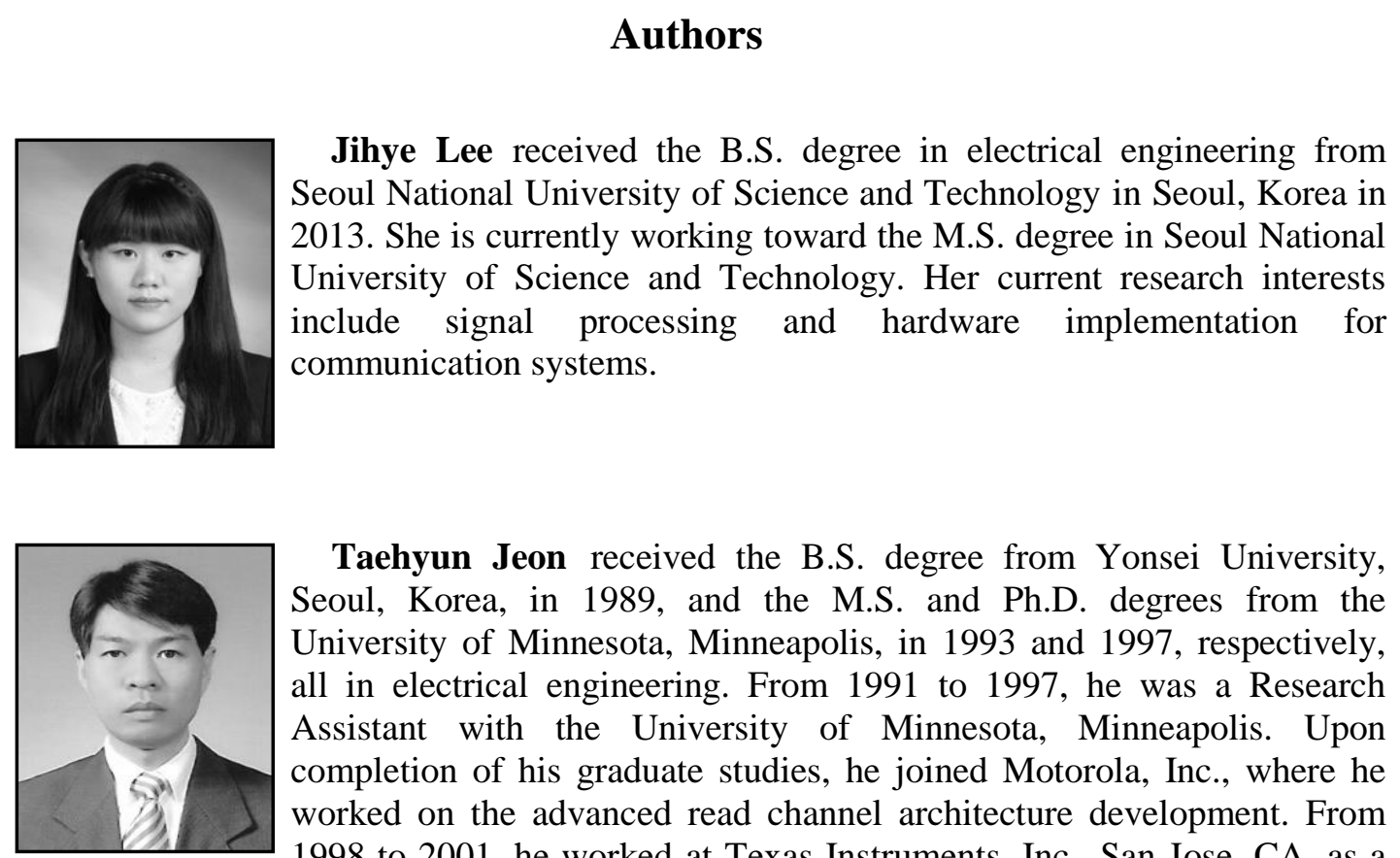

Taehyun Jeon received the B.S. degree from Yonsei University, Seoul, Korea, in 1989, and the M.S. and Ph.D. degrees from the University of Minnesota, Minneapolis, in 1993 and 1997, respectively, all in electrical engineering. From 1991 to 1997, he was a Research Assistant with the University of Minnesota, Minneapolis. Upon completion of his graduate studies, he joined Motorola, Inc., where he worked on the advanced read channel architecture development. From 1998 to 2001, he worked at Texas Instruments, Inc., San Jose, CA, as a member of technical staff. From 2002 to 2005, he worked as a senior member of research staff at the Electronics and Telecommunication Research Institute (ETRI), Daejeon, Korea where he participated in the high capacity wireless local area network system development project. $\mathrm{He}$ is currently a faculty member of the Department of Electrical and Information Engineering at the Seoul National University of Science and Technology in Seoul, Korea. His current research interests include communication theory and signal processing for high capacity wireless and wireline data transmission systems with emphasis on synchronization, signal detection, modulation coding, and the multicarrier and multi antenna systems. 